ARTICLE

\title{
Structural insights into sequence-dependent Holliday junction resolution by the chloroplast resolvase $\mathrm{MOC1}$
}

\author{
Junjie Yan ${ }^{1,2}$, Sixing Hong ${ }^{1,2}$, Zeyuan Guan (iD ${ }^{1}$, Wenjing He ${ }^{1}$, Delin Zhang ${ }^{1} \&$ Ping Yin ${ }^{1 凶}$
}

Holliday junctions (HJs) are key DNA intermediates in genetic recombination and are eliminated by nuclease, termed resolvase, to ensure genome stability. $\mathrm{HJ}$ resolvases have been identified across all kingdoms of life, members of which exhibit sequence-dependent $\mathrm{HJ}$ resolution. However, the molecular basis of sequence selectivity remains largely unknown. Here, we present the chloroplast resolvase $\mathrm{MOC1}$, which cleaves $\mathrm{HJ}$ in a cytosine-dependent manner. We determine the crystal structure of MOC1 with and without HJs. MOC1 exhibits an $\mathrm{RNase} \mathrm{H}$ fold, belonging to the retroviral integrase family. MOC1 functions as a dimer, and the $\mathrm{HJ}$ is embedded into the basic cleft of the dimeric enzyme. We characterize a base recognition loop (BR loop) that protrudes into and opens the junction. Residues from the $B R$ loop intercalate into the bases, disrupt the $C-G$ base pairing at the crossover and recognize the cytosine, providing the molecular basis for sequence-dependent $\mathrm{HJ}$ resolution by $\mathrm{a}$ resolvase.

\footnotetext{
${ }^{1}$ National Key Laboratory of Crop Genetic Improvement and National Centre of Plant Gene Research, Huazhong Agricultural University, 430070 Wuhan, China.

${ }^{2}$ These authors contributed equally: Junjie Yan, Sixing Hong. ${ }^{\circledR}$ email: yinping@mail.hzau.edu.cn
} 
$\mathrm{H}$ olliday junctions (HJs) are a central intermediate formed during the process of homologous recombination ${ }^{1}$, playing a critical role in promoting genetic diversity and repairing double-stranded DNA breaks ${ }^{2}$. HJs exhibit a cross-stranded fourway junction structure involving homologous pairing and DNA strand exchange ${ }^{3,4}$, and must be timely eliminated to maintain faithful chromosome segregation and genome stability ${ }^{5}$. Defective $\mathrm{HJ}$ resolution would lead to aberrant chromosome morphology, resulting in tumorigenesis in humans ${ }^{6,7}$.

One of the key pathways for $\mathrm{HJ}$ resolution is carried out by a family of structure-selective endonucleases called HJ resolvases, which have been identified from a wide variety of organisms including bacteriophages, bacteria, archaea, yeasts, plants, and mammals ${ }^{8-12}$. HJ resolvases have been extensively studied in lower organisms including phage $\mathrm{T} 4$ and $\mathrm{T} 7$ endonucleases ${ }^{13,14}$, bacterial RuvC ${ }^{15}$, and archaeal $\mathrm{Hjc}^{16}$. In eukaryotes, the nucleus-encoded Gen 1 and its orthologs have been broadly investigated $^{6,11,17-20}$. In addition, HJ resolvases have also been identified in mitochondria and chloroplasts, including $\mathrm{Cce}^{21}$, $\mathrm{Ydc}^{22}$, and $\mathrm{MOC}^{23}$.

These resolvases exhibit distinct cleavage characteristics. T4 and T7 endonucleases display no sequence specificity for cleavage. In contrast, sequence-dependent $\mathrm{HJ}$ resolution was reported for RuvC $\left(5^{\prime}-\mathrm{A} / \mathrm{TTT}^{\downarrow} \mathrm{G} / \mathrm{C}-3^{\prime}\right)^{8,15,24}$, Ccel $\left(5^{\prime}-\mathrm{ACT}^{\downarrow} \mathrm{A}-3^{\prime}\right)^{25,26}$, Ydc2 $\left(5^{\prime}-\mathrm{C} / \mathrm{TT}^{\downarrow}-3^{\prime}\right)^{27,28}$, and MOC1 $\left(5^{\prime}-\mathrm{C}^{\downarrow} \mathrm{C}-3^{\prime}\right)^{23}$. Crystal structures of $\mathrm{HJ}$-bound $\mathrm{T} 4$ endo VII and $\mathrm{T} 7$ endo I revealed that the enzymes interact exclusively with the backbone of DNA and form no hydrogen bonds with the DNA bases ${ }^{13,14}$, which explains their lack of sequence specificity. The complex structure of TtRuvC-HJ was obtained in a noncatalytic state and no direct base-recognition was observed ${ }^{29}$, which does not explain the molecular basis of the cleavage specificity. To date, the molecular basis of sequence-dependent $\mathrm{HJ}$ resolution by resolvase remains largely unknown.

In this study, we determine the crystal structures of HJ-free Zea mays MOC1 (ZmMOC1), Nicotiana tabacum MOC1 (NtMOC1), and HJ-bound NtMOC1 at resolutions of $2.5 \AA$, $2.0 \AA$, and $2.5 \AA$, respectively. MOC1 exhibits dimerization and an $\mathrm{RNaseH}$ fold, belonging to the retroviral integrase superfamily. The $\mathrm{HJ}$ is embedded into the basic cleft of the dimeric enzyme. Four acidic residues constitute the catalytic tetrad that acts on the cleavable phosphodiester bond. Moreover, we characterize a base recognition loop (BR loop) that protrudes into the junction and disrupts the $\mathrm{C}-\mathrm{G}$ base pairs at the crossover. Residue D183 from the BR loop recognizes the cytosine, determining the cytosine-dependent $\mathrm{HJ}$ resolution.

\section{Results}

Chloroplast MOC1s exhibit cytosine-dependent HJ resolution. Recently, mutational screening analysis identified the chloroplastlocalized HJ resolvase MOC1 that is essential for chloroplast nucleoid segregation in Chlamydomonas reinhardtii and Arabidopsis thaliana ${ }^{23}$. Dysfunction of MOC1 resulted in aberrant nucleoid morphology, indicating a crucial role for this kind of gene in plant chloroplast development. At the biochemical level, AtMOC1 introduced a cleavage between two consecutive cytosine $\left(5^{\prime}-\mathrm{C}^{\downarrow} \mathrm{C}-3^{\prime}\right)$ residues at the core of the $\mathrm{HJ} \mathrm{s}^{23}$. MOC1 homologs are widely present across the plant kingdom with a highly conserved C-terminal domain (Supplementary Fig. 1). In this study, we purified homologous MOC1s from various plant species including A. thaliana (AtMOC1), Glycine max (GmMOC1), Gossypium raimondii (GrMOC1), N. tabacum (NtMOC1), Oryza sativa (OsMOC1), and Z. mays (ZmMOC1).

To examine the HJ cleavage specificity of these MOC1 orthologs, a synthetic bimobile $\mathrm{HJ}$ of a 2-base-pair (2-bp) homologous core with a CCGG core (Supplementary Fig. 2), termed X2 (CCGG), was used as a control according to a previous study ${ }^{23}$. In addition, six additional X2 variants with mutations in the homologous cores were used as the substrates (Fig. 1a). All these MOC1s exhibited obvious $\mathrm{HJ}$ resolution activity toward the X2 (CCGG) substrate (Fig. 1b), similar to a previous investigation ${ }^{23}$, indicating cleavage between the consecutive cytosine residues $\left(5^{\prime}-C^{\downarrow} \mathrm{C}-3^{\prime}\right)$. In addition, weak cleavage activity against X2 (CGCG) and X2 (CATG) were also observed for some orthologous MOC1s (Fig. 1b). Further cleavage site mapping experiments using AtMOC1 revealed symmetrical cuttings at the crossover in the sequence $5^{\prime}-\mathrm{C}^{\downarrow} \mathrm{G}-3^{\prime}$ for the X2 (CGCG) substrate and $5^{\prime}-\mathrm{C}^{\downarrow} \mathrm{A}-3^{\prime}$ for the X2 (CATG) substrate (Supplementary Fig. 3). These results revealed that MOC1s resolve $\mathrm{HJs}$ in a cytosine-dependent manner (after a cytosine).

Crystal structure of MOC1. To elucidate the molecular mechanism of cytosine-dependent $\mathrm{HJ}$ resolution by MOC1s, we then performed a systematic crystal screening for these homologous MOC1s. We first determined the crystal structure of HJfree ZmMOC1 (T107-V280) via iodide-based single-wavelength anomalous diffraction (I-SAD) at a refined resolution of $2.5 \AA$ (Methods and Supplementary Fig. 4a-b) and the structure of a quadruple mutant NtMOC1 (N108-S275, I112V/Q162K/E235Q/ 239Q) at a $2.0 \AA$ resolution (Fig. 2a, Supplementary Table 1). Structural alignment of NtMOC1 with $\mathrm{ZmMOC1}$ revealed a similar fold with an RMSD value of $0.919 \AA$ (Supplementary Fig. 4c). Moreover, the crystal structures of the HJ-bound NtMOC1 were also determined. Thus, we use NtMOC1 as a representative to demonstrate the structural features of $\mathrm{MOC} 1$ in the following section.

NtMOC1 is a homodimer (Fig. 2b), akin to the known resolvases ${ }^{18,19,30-38}$. For each protomer, the core of NtMOC1 is formed by five-stranded $\beta$-sheets $(\beta 1 \beta 2 \beta 3 \beta 6 \beta 7)$ composed of three antiparallel $(\beta 1 \beta 2 \beta 3)$ and two parallel strands $(\beta 6 \beta 7)-a$ characteristic feature of the RNase $\mathrm{H}$ fold ${ }^{39}$, belonging to the retroviral integrase superfamily ${ }^{39}$. The $\beta$-sheets are flanked by six $\alpha$ helices ( $\alpha 1$ and $\alpha 2$ on one side, and $\alpha 3, \alpha 4, \alpha 5$ and $\alpha 6$ on the other side) (Fig. 2b, c). The $a$-helix 2 ( $a 2$ ) from each protomer constitutes a helical bundle, where G192, G196, G200, and A204 form a hydrophobic patch on the dimer interface (Fig. 2d, e; Supplementary Fig. 5a). Both analytical ultracentrifugation (AUC) and sizeexclusion chromatography (SEC) experiments revealed that double mutations of G200E and A204E could disrupt dimer formation (Supplementary Fig. 5b, c). The SEC results further indicate that single mutation of G200E or A204E might partially disrupt the dimerization (Supplementary Fig. 5c). Although these mutants retain some $\mathrm{HJ}$-binding ability, their $\mathrm{HJ}$ cleavage activity is drastically impaired (Supplementary Fig. 5d, e), demonstrating that NtMOC1 functions as a dimer.

Both protomers of apo NtMOC1 revealed identical architectures with no structural variation (Supplementary Fig. 6a). A structural homolog search by $\mathrm{Dali}^{40}$ revealed that NtMOC1 has the highest structural similarity with RuvC resolvase (Supplementary Table 2). In addition, NtMOC1 displays some similarity to Cas9, due to the existence of a RuvC nuclease domain in the protein ${ }^{41}$. Structural superimposition of NtMOC1 with RuvC revealed similar protomers (with an RMSD value of $3 \AA$ ) and dimeric architectures (Supplementary Fig. $6 \mathrm{~b}, \mathrm{c}$ ), suggesting their potentially similar $\mathrm{HJ}$ binding and resolution activities.

Crystal structure of NtMOC1 in complex with HJ. To determine the complex structure, a series of HJs with varying arm lengths and homologous cores were used for cocrystallization 
a

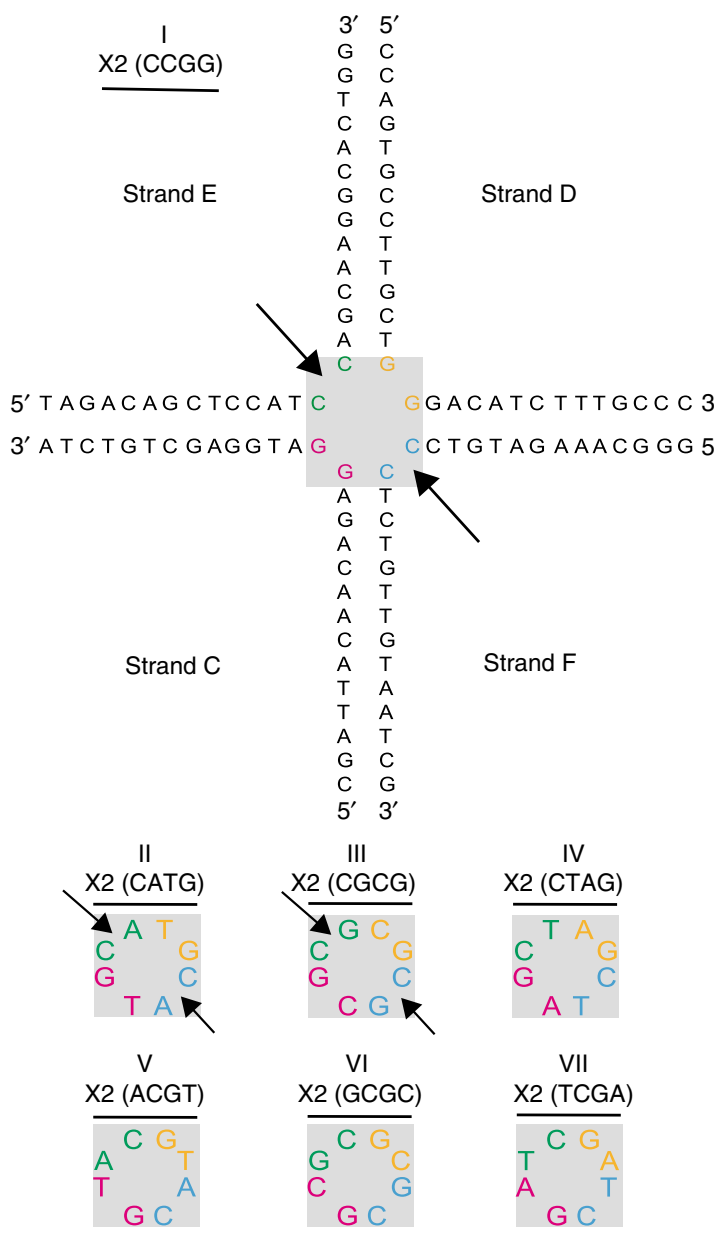

b
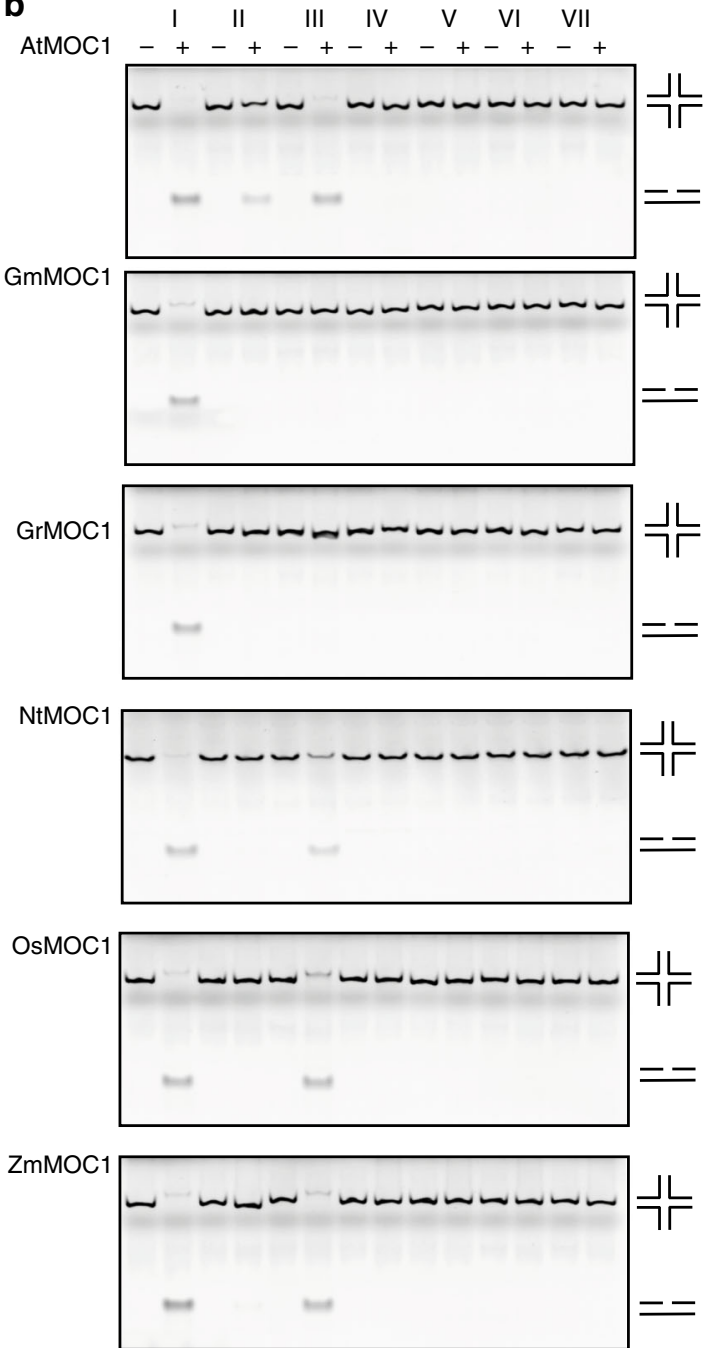

Fig. 1 Sequence-dependent HJ cleavage by chloroplast MOC1s. a Schematic drawing of the HJ substrate X2. X2 was prepared by annealing 4 DNA oligos. The upper panel shows the structure of X2 (CCGG). Two homologous base pairs are shaded in gray. The lower panel indicates X2s with variant CCGG cores. These X2s share identical nucleotides as X2 (CCGG) except the 2 bp homologous cores. These seven X2s are numbered sequentially (I-VII). Arrows indicate the cleavage site. $\mathbf{b}$ The sequence-specific $\mathrm{HJ}$ resolution activity of MOC1 orthologs was examined. AtMOC1, GmMOC1, GrMOC1, NtMOC1, OsMOC1, and ZmMOC1 are MOC1s from Arabidopsis thaliana, Glycine max, Gossypium raimondii, Nicotiana tabacum, Oryza sativa, and Zea mays, respectively. The final $\mathrm{HJ}$ concentration in each lane is $250 \mathrm{nM}$. The final protein concentration of $\mathrm{MOC} 1$ is $1000 \mathrm{nM}$. The reactions were examined by native PAGE and visualized by GelRed staining. Labels on the top indicate the different X2 substrates used as shown in $\mathbf{a}$. Source data are provided as a Source Data file.

with MOC1s. Most crystals were either poorly diffracted or showed no DNA density after data processing. After tedious trials, we finally determined two crystal structures of the NtMOC1-HJ complex at an approximately $2.5 \AA$ resolution in the $\mathrm{P} 2{ }_{1} 2{ }_{1} 2$ space group (Supplementary Table 1). One of the structures contains the active NtMOC1 and $\mathrm{HJ}$ with a noncognate CATG core sequence, and the other is composed of a cleavageinactive NtMOC1 mutant (D116A/E175A/D253A/E258A) and an HJ with a cognate CCGG core sequence (Supplementary Fig. 7). The HJs in both structures are four-stranded with a 9-bp arm length in each direction with only variation in the crossover with either the CATG core or the CCGG core (Supplementary Fig. 8a, b). These two NtMOC1-HJ complexes exhibit a nearly identical overall fold (Supplementary Fig. 8c). The present and previous $^{23}$ cleavage site mapping experiments revealed the phosphodiester bond within the CC or CA bases at the crossover is cleavable (Supplementary Fig. 2). In the HJs of both complexes, the CATG and CCGG at the branch point were well aligned (Supplementary Fig. 8d).
Here, we use the complex structure of NtMOC1-HJ with a CATG core sequence as a representative to demonstrate the catalytic and binding features. NtMOC1 in the complex also exhibits dimerization, and the $\mathrm{HJ}$ is embedded into the cleft of the dimeric NtMOC1 (Fig. 3a). Structural alignment of the apo NtMOC1 with HJ-bound NtMOC1 revealed minor conformational changes with an RMSD value of $0.527 \AA$ over $242 \mathrm{Ca}$ (Supplementary Fig. 9), which mainly arose from the loop preceding a2 (we termed this loop the base recognition loop (BR loop), discussed below). An additional pair of unique antiparallel $\beta$-sheets $(\beta 4 \beta 5)$ was observed only in the complex structure flanking the dimer interface but the density corresponding to these residues in the apo structure is missing (Fig. 2b and Fig. 3a), suggesting a role for this segment in DNA interactions.

Catalytic center of NtMOC1. In the HJ-bound complex structure, HJ exhibits an open planar and X-shaped conformation with an overall two-fold symmetry (Fig. 3b). The phosphate backbones of the non-exchanging strands are antiparallel, whereas the 
a

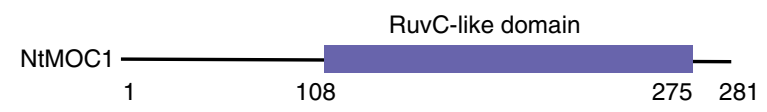

b

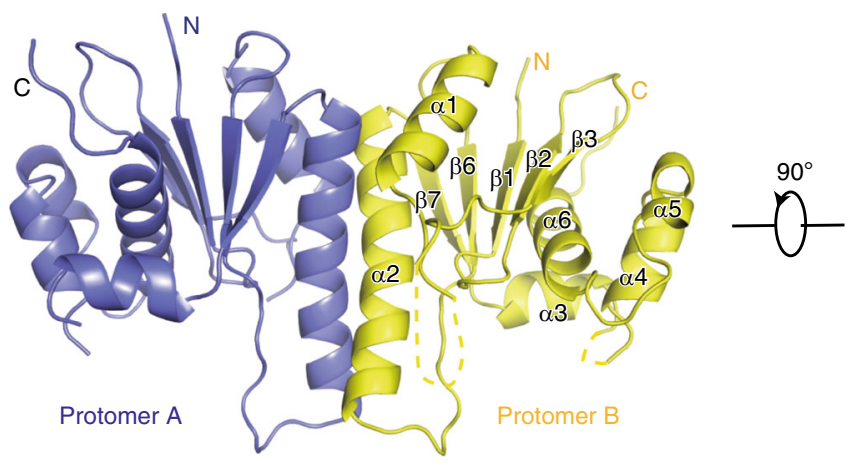

C

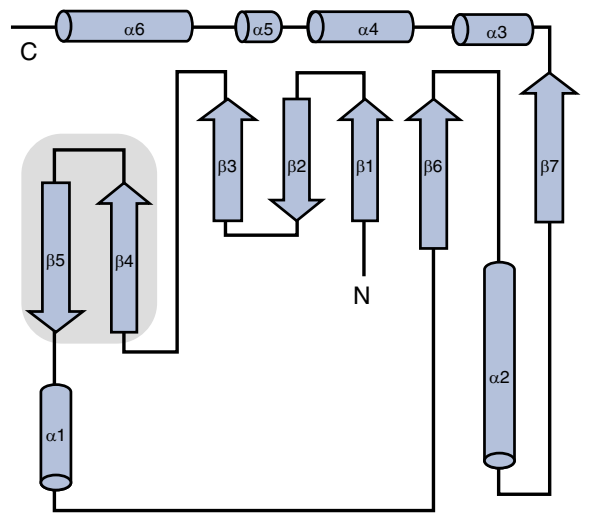

d

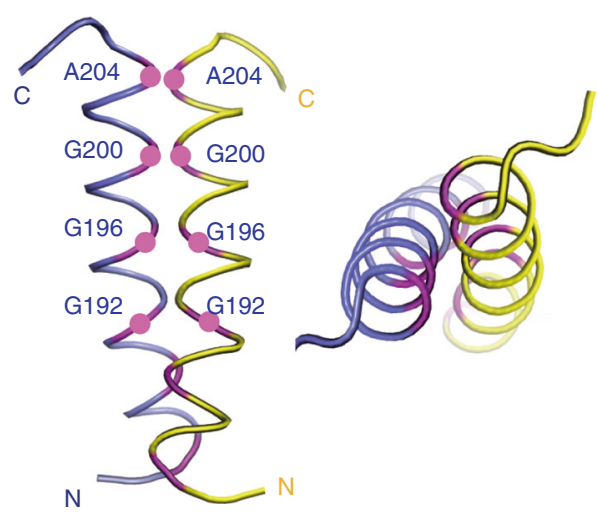

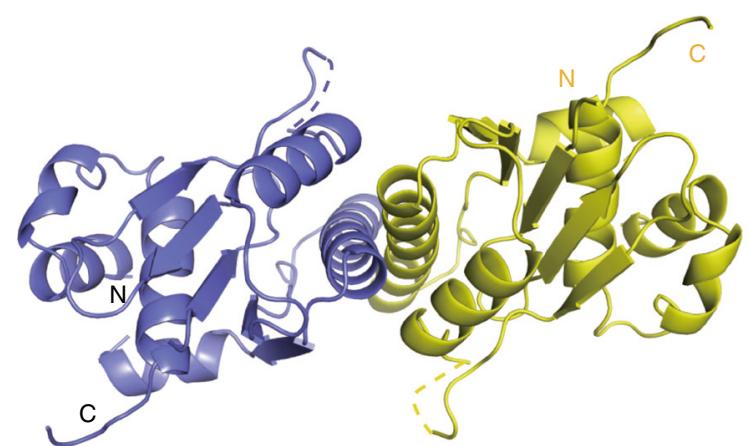

e

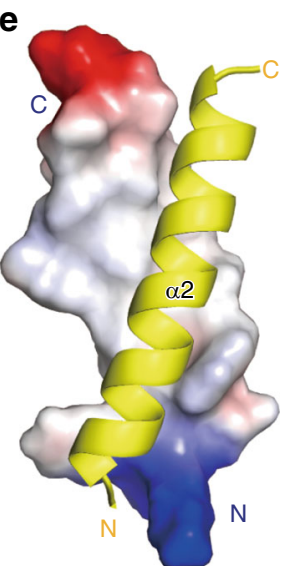

Fig. 2 Crystal structure of apo NtMOC1. a Schematic domain structure of NtMOC1. The C-terminus (residues 108-275) is the RuvC-like domain, the structure of which was determined in this study. $\mathbf{b}$ Two perpendicular views of the overall structure of apo NtMOC1. Secondary structural elements are labeled in protomer B. Dashed lines indicate residues with missing electron density. c Topological diagram of the NtMOC1 protomer. Secondary structure elements are labeled as in $\mathbf{a}$. Two $\beta$-sheets $(\beta 4-\beta 5)$ observed in the complex structure are shaded in gray. The electron density of this segment is missing in the apo structure. $\mathbf{d}$ Residues with small side chains constitute the dimer interface. $\mathbf{e}$ Dimer interface of the helix bundle. $\alpha 2 \mathrm{~s}$ from protomers $\mathrm{A}$ and $\mathrm{B}$ are shown in the electrostatic surface potential and in the yellow cartoon illustration, respectively. The blue and red colors indicate positively and negatively charged residues, and white regions indicate hydrophobic residues.

phosphate backbones of the exchanging strands are sharply kinked at the crossover to form a U-turn. The center of the junction is opened by NtMOC1 into a parallelogram with cross dimensions of $32 \AA$ between the non-exchanging strands and $9 \AA$ between the exchanging strands (Fig. 3b). The scissile phosphates are in close proximity to the two active sites of the dimeric enzyme (Supplementary Fig. 10a). Four acidic amino acids, namely D116, D118, E175, and E258, coordinate a magnesium ion approach to the cleavable phosphodiester backbone, constituting the catalytic tetrad (Fig. 3c). Alanine substitutions of D116, D118, E175 and E258 completely abolished $\mathrm{HJ}$ cleavage activity, although the $\mathrm{HJ}$ binding activity was retained (Fig. 3d, e). As a control, mutation of residue D253 neighboring the active site showed no effect on either $\mathrm{HJ}$ binding or $\mathrm{HJ}$ cleavage activity compared with the wild-type NtMOC1 (Supplementary Fig. 10b-d).

Protein-DNA contacts. The complex structure reveals extensive interactions between dimeric NtMOC1 and four DNA strands with a global contact area of $2595 \AA^{2}$ (Fig. 4a, Supplementary Fig. 11). To clearly demonstrate the protein-DNA interactions, we defines the four DNA strands (C, D, E and F). Each strand harbors 18 nucleotides numbered sequentially from the $5^{\prime}$ side. The arm length in each direction is $9 \mathrm{bp}$ (Supplementary
Fig. 8a-b), and the phosphodiester to be cleaved is located at the crossover between cytosine 9 (C9) and adenine 10 (A10) at the continuous strands $\mathrm{E}$ and $\mathrm{F}$ based on the cleavage site mapping ${ }^{23}$ (Supplementary Fig. 2), complex structure alignment (Supplementary Fig. 8), and its proximity to the catalytic tetrad (Fig. 3c).

Electrostatic potential analysis revealed that a high proportion of positively charged amino acids are distributed on the surface toward the HJ (Fig. 4a). The residue R149 in $\beta 5$ makes direct contacts with the neighboring DNA strands (Fig. 4b). The amine group of R149 (protomer B) interacts with the phosphate group of nucleotides G12 (chain E) and A12 (chain D) (Fig. 4b). The K185 residues from both protomers are located at the $\mathrm{N}$-terminus of the dimeric helix bundle, protruding into the junction, interacting with the phosphate group of the flipped-out nucleotide G10 from the non-exchanging strands (Fig. 4c). K218 (protomer A) from $\alpha 5$ contacts the phosphate group of nucleotide $\mathrm{C} 9$ (chain F), and the neighbored K225 (Protomer A) contacts the phosphate group of nucleotide C8 (chain F) via its main chain amine group (Fig. 4d). Three consecutive basic residues (R250/K251/K252) at the periphery of the dimeric enzyme contact the exchanging DNA strands (Fig. 4e). These interactions were verified by mutational analysis. Charge reversal mutants with single mutations of these residues (R149D, K185D, and $\mathrm{K} 218 \mathrm{D}$ ) retained equivalent $\mathrm{HJ}$ binding activity (Supplementary Fig. 12a). However, the HJ 
a

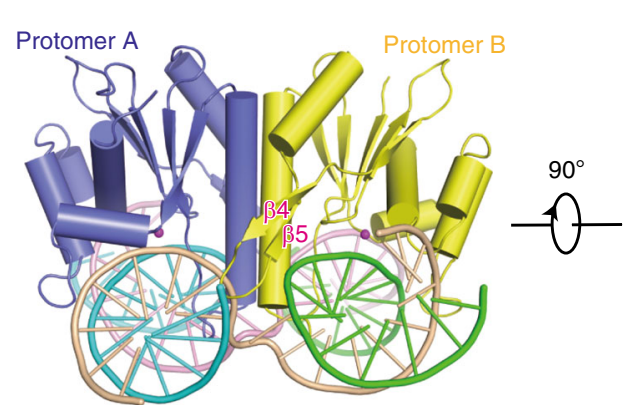

C

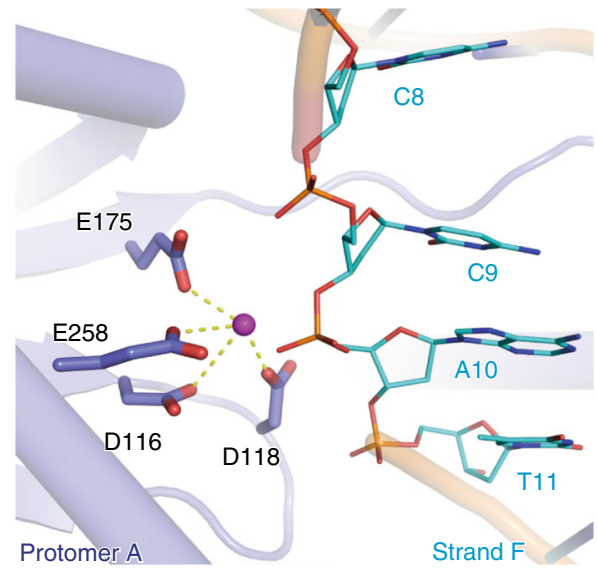

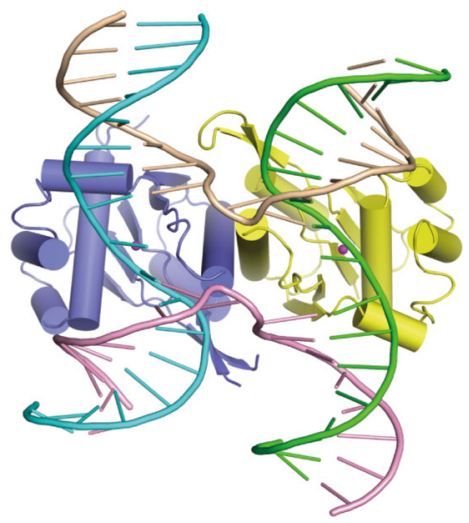

b

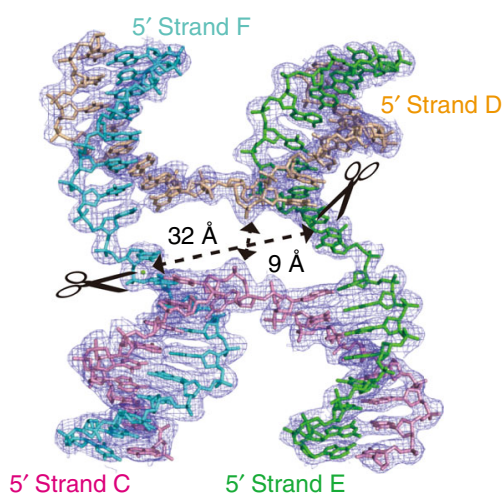

d

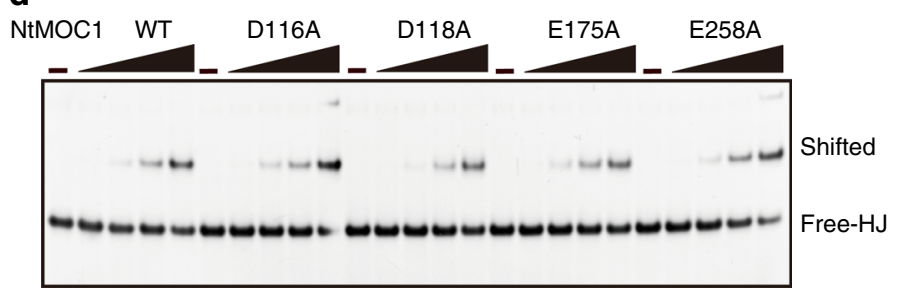

e

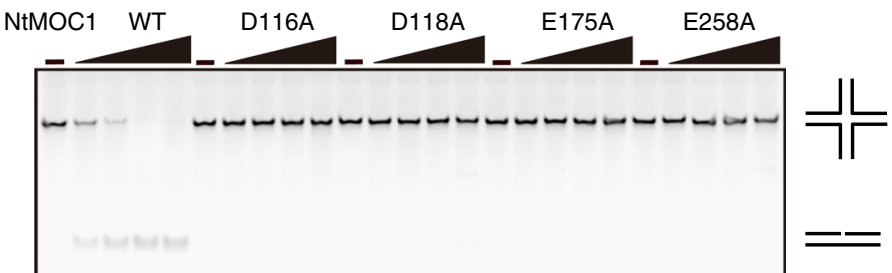

Fig. 3 Crystal structure of NtMOC1 in complex with a synthetic HJ. a Two perpendicular views of the complex structure. Subunits of the protein dimer are shown in slate and yellow. The four DNA strands C, D, E, and F are colored in pink, wheat, cyan, and green, respectively. The HJ is embedded into the cleft of the dimeric enzyme. $\mathbf{b}$ Structure of the $\mathrm{HJ}$ in the complex. The electron density map is contoured at $1.0 \mathrm{\sigma}$. Scissors indicate the scissile phosphates. Distances between the exchanging and non-exchanging strands are measured. c Active centers of NtMOC1. The magnesium ions are shown as purple spheres. Four acidic amino acids, coordinating with magnesium, constitute the catalytic tetrad. $\mathbf{d}$ Mutations at the catalytic tetrad of NtMOC1 have no effect on the $\mathrm{HJ}$ binding activity. e Mutations at the catalytic tetrad of $\mathrm{NtMOC} 1$ abolish the $\mathrm{HJ}$ cleavage activity. For the $\mathrm{HJ}$ binding and cleavage assay, $\mathrm{X} 2$ (CCGG) was used as $\mathrm{HJ}$ substrate. The final concentration of $\mathrm{HJ}$ in each lane was $250 \mathrm{nM}$. Five gradients with increasing concentrations $(0,125,250,500$, and $1000 \mathrm{nM}$ ) were applied for each protein sample. The reactions were resolved by native PAGE and visualized by GelRed staining. Source data are provided as a Source Data file.

cleavage activity of R149D, K185D and K218D was completely lost (Supplementary Fig. 12b), suggesting that these three residues are essential for maintenances of the $\mathrm{HJ}$ in a favorable configuration for cleavage. The control mutant $\mathrm{K} 225 \mathrm{D}$ retains the $\mathrm{HJ}$ cleavage activity (Supplementary Fig. 12b). Simultaneous mutation of R149D/K185D/K218D/K225D (Tetra-M) or R250/K251/K252D could result in total loss of $\mathrm{HJ}$ binding and cleavage activity (Supplementary Fig. 12).

Molecular basis of cytosine-dependent $\mathrm{HJ}$ resolution by MOC1. Close inspection of the HJ-bound structure revealed how MOC1 cleaved $\mathrm{HJ}$ in a cytosine-dependent manner $\left(5^{\prime}-\mathrm{C}^{\downarrow} \mathrm{C} / \mathrm{G}-3^{\prime}\right)$. Two amino acids (Y180 and D183) in the loop preceding $\alpha 2$ are involved in sequence-specific $\mathrm{HJ}$ resolution (Supplementary Fig. 13). Hereafter, we refer to this loop as the base recognition loop (BR loop). Residue Y180 intercalates into the bases one nucleotide prior to the cleavage site (Fig. 5a), forming stacking interactions with the flanking nucleotide bases, similar to the aromatic residue $\mathrm{F} 69$ observed in the corresponding loop of E.coli RuvC (F73 in T. thermophilus) ${ }^{29,42,43}$. More importantly, D183 specifically recognizes the cytosine (C9) via a hydrogen bond (Fig. 5a). Alanine substitution of D183 (D183A) results in complete abolishment of $\mathrm{HJ}$ cleavage activity, although the $\mathrm{HJ}$ binding activity is only partially decreased (Fig. 5b, c). Mutation of Y180 (Y180A) has little effect on HJ binding and resolution activity (Fig. 5b, c). Although the overall structures of apo and HJ-bound NtMOC1 exhibited similar architectures (Supplementary Fig. 9), residues Y180 and D183 in the BR loop underwent striking conformational changes, demonstrating the great flexibility of the BR loop (Fig. 5d).

The pairing of D183 (protomer A) to the cytosine $(\mathrm{C} 9$, from strand F) led to flipping out of the initially paired guanine (G10, from strand D) from the duplex (Fig. 5a, Supplementary Fig. 12). The flipped-out guanine (G10) is further stabilized by hydrogen bond networks with amino acids Q186 and G187 of helix 2 (a2) from protomer $\mathrm{B}$ and the surrounding nucleotides (Fig. 5a). This MOC1-induced guanine flipping is reminiscent of other base flipping phenomena including epigenetic regulation of DNA and base excision repair by some modification-related nucleases, such as HhaI methyltransferase ${ }^{44}$ and uracil-DNA glycosylase ${ }^{45}$.

Based on the structural and biochemical results, we propose a working model of MOC1 (Fig. 6). In general, HJ forms a compact structure with all base pairings 3,4 , but is distorted when the dimeric MOC1 penetrates into the junction and disrupts the $\mathrm{C}-\mathrm{G}$ 


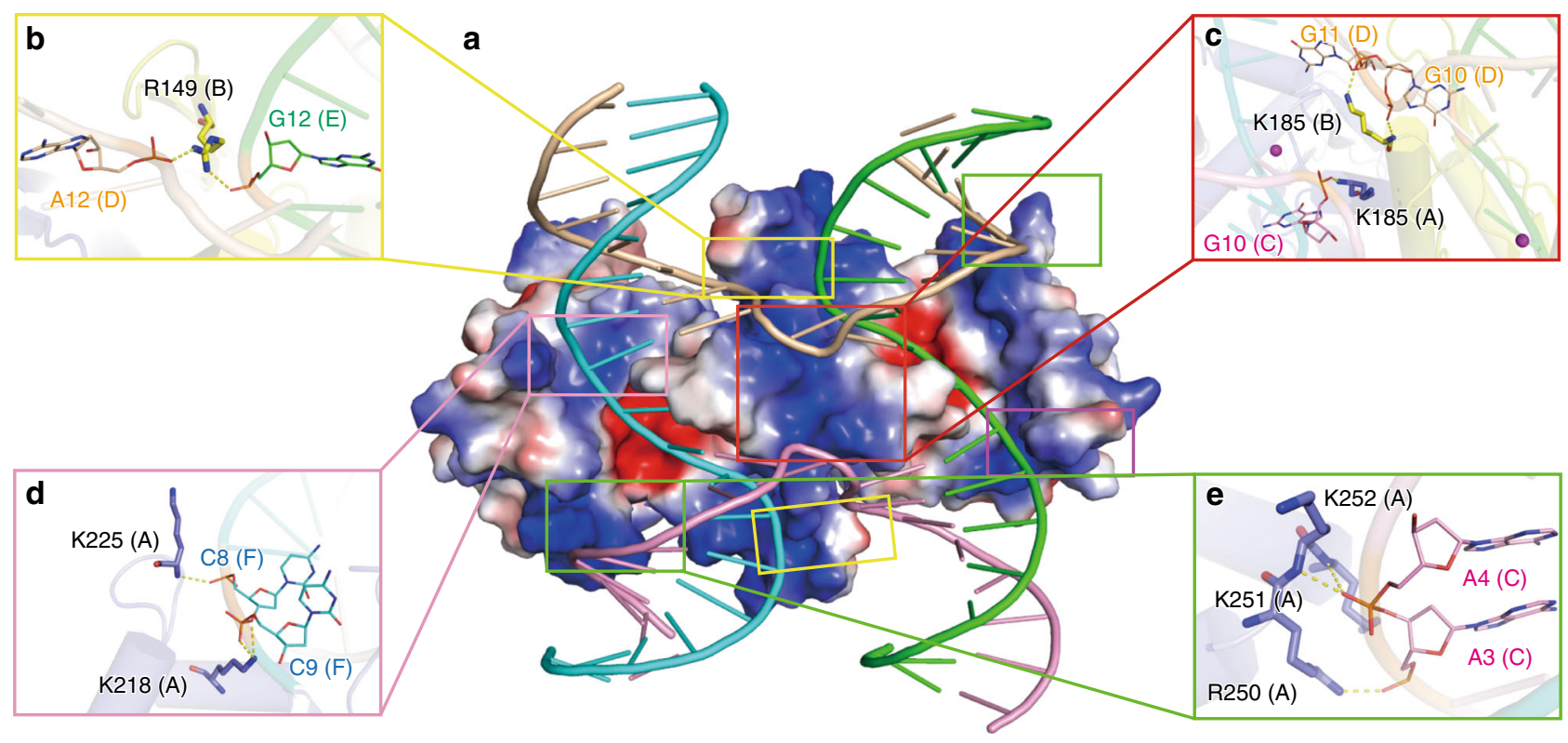

Fig. 4 Protein-DNA contacts. a The bottom view of the complex shows the interaction surface. The protein dimer is shown by the electrostatic surface potential. Four DNA strands of the $\mathrm{HJ}$ are shown with cartoon illustrations. Four distinct interaction regions are labeled with colored rectangles. b-e Enlarged image showing the details of the interaction between NtMOC1 and the DNA strands. Capital letters in parentheses indicate corresponding protein or nucleotide chains. A and B represent protomer A and B; C, D, E, and F represent DNA strands C, D, E and F, respectively.

base pairing at the crossover prior to the cleavage-site. To fulfill sequence-dependent cleavage, D183 in the BR loop specifically recognizes the cytosine via a hydrogen bond, favoring hydrolysis of the phosphodiester bond by the catalytic tetrad in a cytosinedependent manner.

\section{Discussion}

HJs are important DNA intermediates in DNA damage repair and genetic recombination processes, and must be timely resolved into duplex DNA to maintain genome stability. Several kinds of resolvases possess the ability to resolve the $\mathrm{HJ}$ in a sequencespecific manner. HJs are highly dynamic structures that could undergo conformer exchange and branch migration, the relationship between $\mathrm{HJ}$ dynamics and sequence-specific resolution is largely unknown. Using single-molecule fluorescence resonance energy transfer (smFRET), a recent study indicated that HJs remain dynamic in the presence of resolvases (as exemplified by T7 endo I, RuvC, GEN1, and hMus81-Eme1) ${ }^{46}$. Due to the multivalent interactions between the dimeric enzymes and $\mathrm{HJs}$, the resolvase-HJ complex could go through a partially dissociated intermediate state whereby the dynamic process of conformer exchange and branch migration can proceed without full dissociation ${ }^{46}$. In this study, we present the crystal structures of apo and HJ-bound chloroplast resolvase MOC1 and identify a crucial $\mathrm{BR}$ loop in MOC1 that contributes to the sequence-dependent $\mathrm{HJ}$ resolution. We speculate that the presence of MOC1 might not restrain the dynamics of the $\mathrm{HJ}$, which can undergo branch migrations, extending or shortening the length of DNA heteroduplex. Once the cognate sequence of CCGG (or CGCG) moves to the branch point, residues Y180 and D183 from the dynamic BR loop of NtMOC1 could penetrate into the junction and disrupt the $\mathrm{C}-\mathrm{G}$ base pairs. Meanwhile, the disrupted cytosine is recognized by $\mathrm{D} 183$, generating a favorable $\mathrm{HJ}$ configuration, thus facilitating the hydrolysis of the phosphodiester bond by the catalytic tetrad of NtMOC1 (Fig. 6 and Supplementary Fig. 14a).

Previous studies indicated that RuvC $\left(5^{\prime}-\mathrm{A} / \mathrm{TTT}^{\downarrow} \mathrm{G} / \mathrm{C}-3^{\prime}\right)^{8,15,24}$, Ydc2 $\left(5^{\prime}-\mathrm{C} / \mathrm{TT} \mathrm{T}^{\downarrow}-3^{\prime}\right)^{27,28}$, and Ccel $\left(5^{\prime}-\mathrm{ACT} \downarrow^{\downarrow} \mathrm{A}-3^{\prime}\right)^{25,26}$ are all sequence-specific resolvases that introduce a nick after a thymine.
Crystal structures of $\mathrm{RuvC}^{29,30,43}$ and $\mathrm{Ydc}^{33}$ revealed their RNase $\mathrm{H}$ fold, belonging to the retroviral integrase family. Furthermore, these proteins function as dimers, harboring a cleft in each protomer that accommodates binding duplex DNA. Biochemical studies suggested that the presence of RuvC or Ydc2 should disrupt the base pairing of $\mathrm{HJ}$ at the crossover $15,47,48$, as evidenced by the MOC1 structure. A similar long loop is present preceding the $a$-helix that forms a helical bundle in RuvC and Ydc2 (Supplementary Fig. 14b). Moreover, the loop contains a consensus sequence: $\mathrm{E}(\mathrm{X})_{2-4} \mathrm{Y} / \mathrm{F}(\mathrm{X})_{1-2} \mathrm{D} / \mathrm{K} / \mathrm{R} / \mathrm{N} / \mathrm{Q} / \mathrm{S}$. The catalytic glutamate is located at the tip of the loop (Supplementary Fig. 14c). Within the loop, aromatic residues such as tyrosine or phenylalanine are generally present (Supplementary Fig. 14d). Following the aromatic residue (Tyr or Phe), there are charged (Asp, Lys, Arg) or polar (Asn, Gln, Ser) residues (Supplementary Fig. 14d), which might mediate specific base recognition. Thus, we speculated that these resolvases might adopt a similar mechanism by directly recognizing the thymine via certain residues to establish sequence-specific $\mathrm{HJ}$ resolution similar to the activity of MOC1.

During the submission and review of our manuscript, two related works regarding sequence-specific $\mathrm{HJ}$ resolution were reported. In one study, the complex structure of DNA-bound TtRuvC at $3.4 \AA$ was reported ${ }^{49}$. Via molecular dynamics simulations, disruption of the scissile T-A base pairs at the crossover and the flipping out of adenine was observed ${ }^{49}$. Meanwhile, residue R76 was identified to form either stacking or H-bond interactions with the bases of the disrupted T-A base pair. This observation is consistent with our speculation regarding the crucial role of the BR loop in base recognition, as R76 is located in the BR loop (Supplementary Fig. 14d). Another study presented the crystal structures of apo and HJ-bound $Z m M O C 1^{50}$. The structure of $\mathrm{ZmMOC1}$ in this study is nearly identical to the recently reported ZmMOC1 structure (PDB: 6IS9) with an RMSD of $0.45 \AA$ (Supplementary Fig. 15a). Although the HJs were annealed using different sequences and strategies (4 oligos in our study and 2 oligos with a T-loop in the other study), superimposition of the complex structure of NtMOC1-HJ and 
a
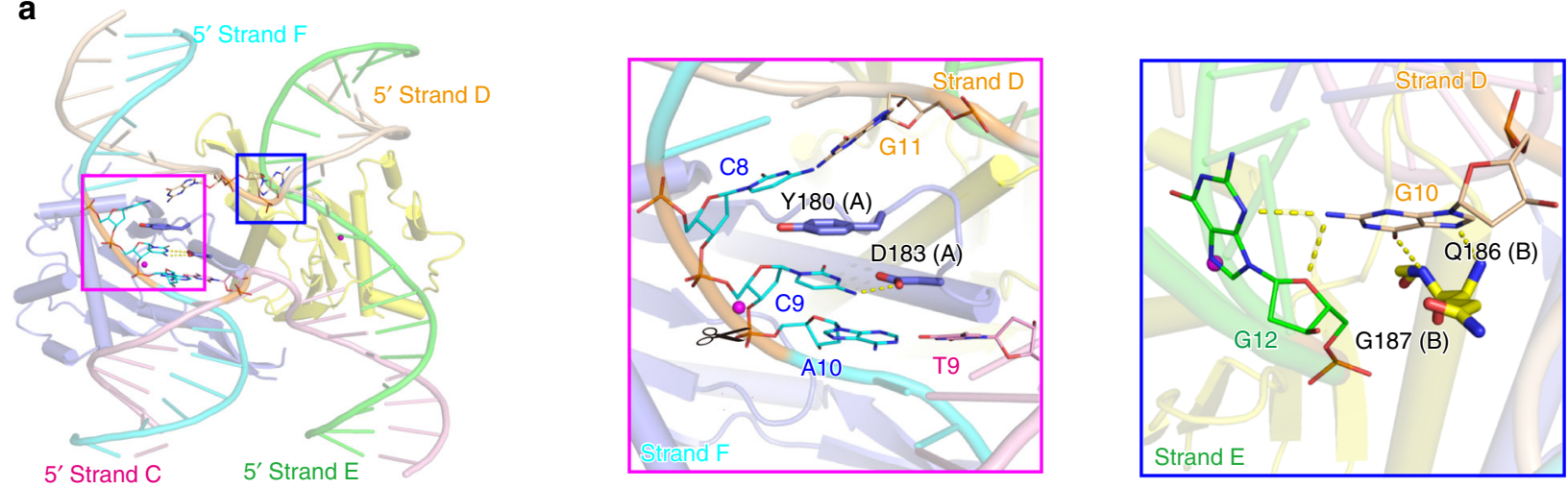

b

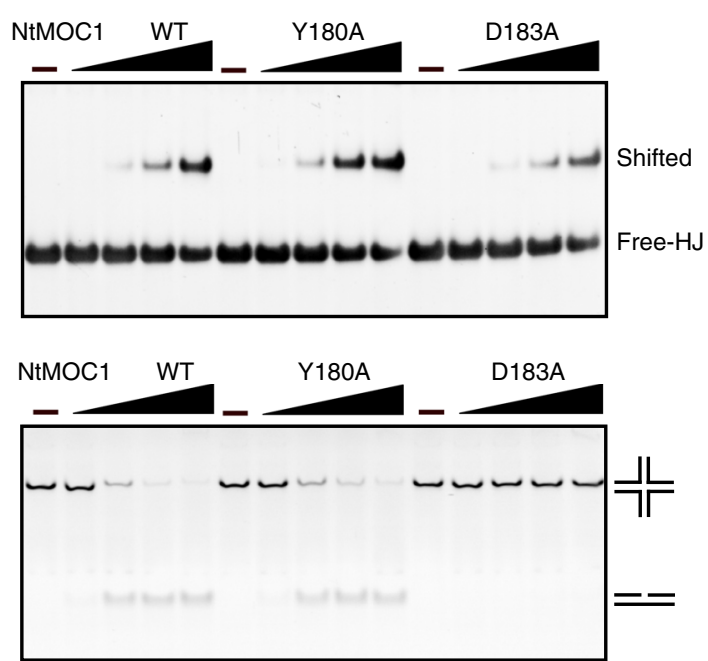

d

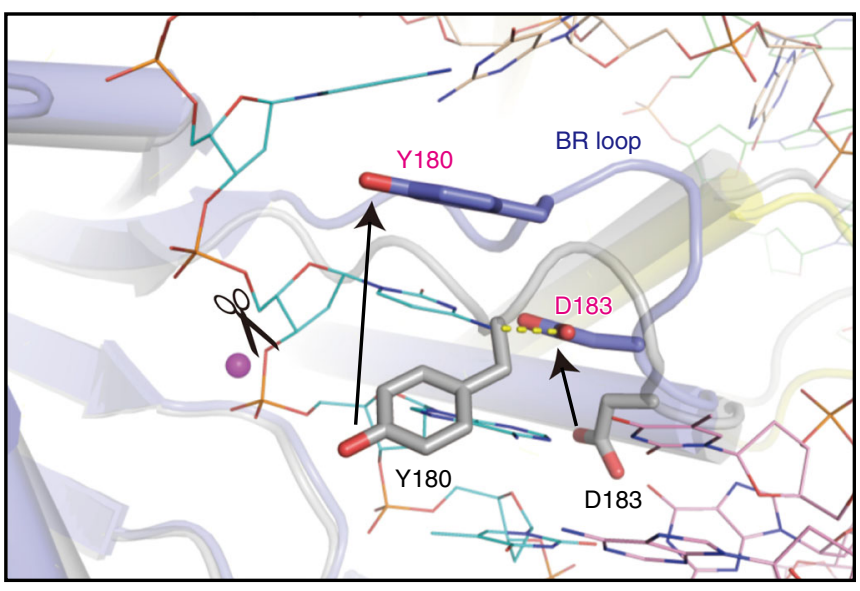

Fig. 5 Molecular basis of cytosine-dependent HJ resolution by NtMOC1. a The C-G base pairs at the crossover are disrupted by NtMOC1. Left panel: Overall view of the disrupted C-G base pairs in the complex. Middle panel: The disrupted cytosine (C9) interacts with D183 via a hydrogen bond. Right panel: The flipped-out guanine is stabilized by residues and nucleotides. b, $\mathbf{c}$ HJ binding (b) and cleavage (c) activity examined for NtMOC1 with alanine substitutions of Y180 and D183. For the HJ binding and cleavage assay, X2 (CCGG) was used as HJ substrate. The final concentration of HJ in each lane was $250 \mathrm{nM}$. Five gradients with increasing concentrations (0, 125, 250, 500, and $1000 \mathrm{nM}$ ) were applied for each protein sample. The reactions were resolved by native PAGE and visualized by GelRed staining. Source data are provided as a Source Data file. d The BR loop undergoes a striking conformational change. Apo NtMOC1 is shown in the gray cartoon illustration. The protomers of HJ-bound MOC1 are shown in slate and yellow cartoons, respectively. DNA strands are shown with lines. The purple circle indicates the magnesium ion in the complex structure. The yellow dashed line indicates the hydrogen bond formed between D183 and cytosine. Arrows indicate the displacement of Y180 and D183. Residues in the apo and complex structures are labeled in black and magenta, respectively. The scissors indicate the cleavage site.

ZmMOC1-HJ exhibited comparable folds with an RMSD of $0.762 \AA$, suggesting a similar DNA recognition mode (Supplementary Fig. 15b). The C-G base pairs at the crossover of these HJs are similarly disrupted (Supplementary Fig. 15c). Moreover, the critical residues Y180 and D183 in the BR loop (BR motif) of $\mathrm{NtMOC} 1$ and the corresponding residues F179 and D182 in ZmMOC1 could be perfectly superimposed (Supplementary Fig. 15d), suggesting a general molecular basis for sequencespecific $\mathrm{HJ}$ resolution by the MOC1 orthologs.

Collectively, our work on NtMOC1 and studies by other groups' on $\mathrm{RuvC}^{49}$ and $\mathrm{ZmMOC1}^{50}$ could serve as important foundations for understanding sequence-specific $\mathrm{HJ}$ resolution by other as-yet-unidentified resolvases.

\footnotetext{
Methods

Molecular cloning, protein expression, and purification. Homologous MOC1s from Arabidopsis thaliana (AT2G26840.1), Glycine max (XP_003532877.2), Gossypium raimondii (XP_012444423.1), Nicotiana tabacum (XP_016490038.1), Oryza sativa (LOC_Os01g16340.1), and Zea mays (ONM31293.1) were cloned into a modified pET15D vector with a $6 \times$ His tag fused at the $\mathrm{N}$ terminus. The DNA mutants were constructed by overlapping PCR. All the constructs were verified by
}

DNA sequencing. The plasmid was transformed into E. coli BL21 (DE3). One litre lysogeny broth medium supplemented with $100 \mathrm{mg} \mathrm{ml}^{-1}$ ampicillin was inoculated with a transformed bacterial pre-culture and shaken at $37^{\circ} \mathrm{C}$ until the cell density reached an $\mathrm{OD}_{600}$ of approximately $1.0-1.2$, protein expression was induced with $0.2 \mathrm{mM}$ isopropyl- $\beta$-D-thiogalactoside at $16^{\circ} \mathrm{C}$ for $12-16 \mathrm{~h}$. The cells were collected by centrifugation, homogenized in buffer A ( $25 \mathrm{mM}$ Tris- $\mathrm{HCl}, \mathrm{pH} 8.0,150 \mathrm{mM}$ $\mathrm{NaCl}$ ), and lysed using a high pressure cell disrupter (JNBIO, China). Cell debris was removed by centrifugation at $20,000 \times g$ for $1 \mathrm{~h}$ at $4{ }^{\circ} \mathrm{C}$, and the supernatant was loaded onto a column equipped with $\mathrm{Ni}^{2+}$ affinity resin (Ni-NTA, Qiagen), washed with buffer B ( $25 \mathrm{mM}$ Tris- $\mathrm{HCl}, \mathrm{pH} 8.0,150 \mathrm{mM} \mathrm{NaCl}, 15 \mathrm{mM}$ imidazole), and eluted with buffer C (25 mM Tris-HCl, pH 8.0, $500 \mathrm{mM} \mathrm{NaCl}, 250 \mathrm{mM}$ imidazole). The $6 \times$ His tag was removed through DrICE digestion. The protein was two-fold diluted to reduce the salt concentration and then separated by HiTrap Heparin (GE Healthcare) using a linear $\mathrm{NaCl}$ gradient in buffer $\mathrm{A}$. The purified protein was concentrated and subjected to gel filtration chromatography (Superdex-200 Increase 10/300, GE Healthcare) in a buffer containing $25 \mathrm{mM}$ Tris- $\mathrm{HCl}$, $\mathrm{pH} 8.0,500 \mathrm{mM} \mathrm{NaCl}$, and $5 \mathrm{mM}$ dithiothreitol. Purity of the proteins was examined using SDS-PAGE and visualized by Coomassie blue staining through all purification processes. The peak fractions were collected and stored at $-80{ }^{\circ} \mathrm{C}$. The mutant proteins were purified similarly as the wild-type proteins.

DNA sample preparation. The sequences of the oligo-nucleotides used to form the HJ substrates were synthesized (TianYi HuiYuan). To make the X2 (CCGG) HJ, the following four DNA strands (5'-GGGCAAAGATGTCCCTCTGTTGT 


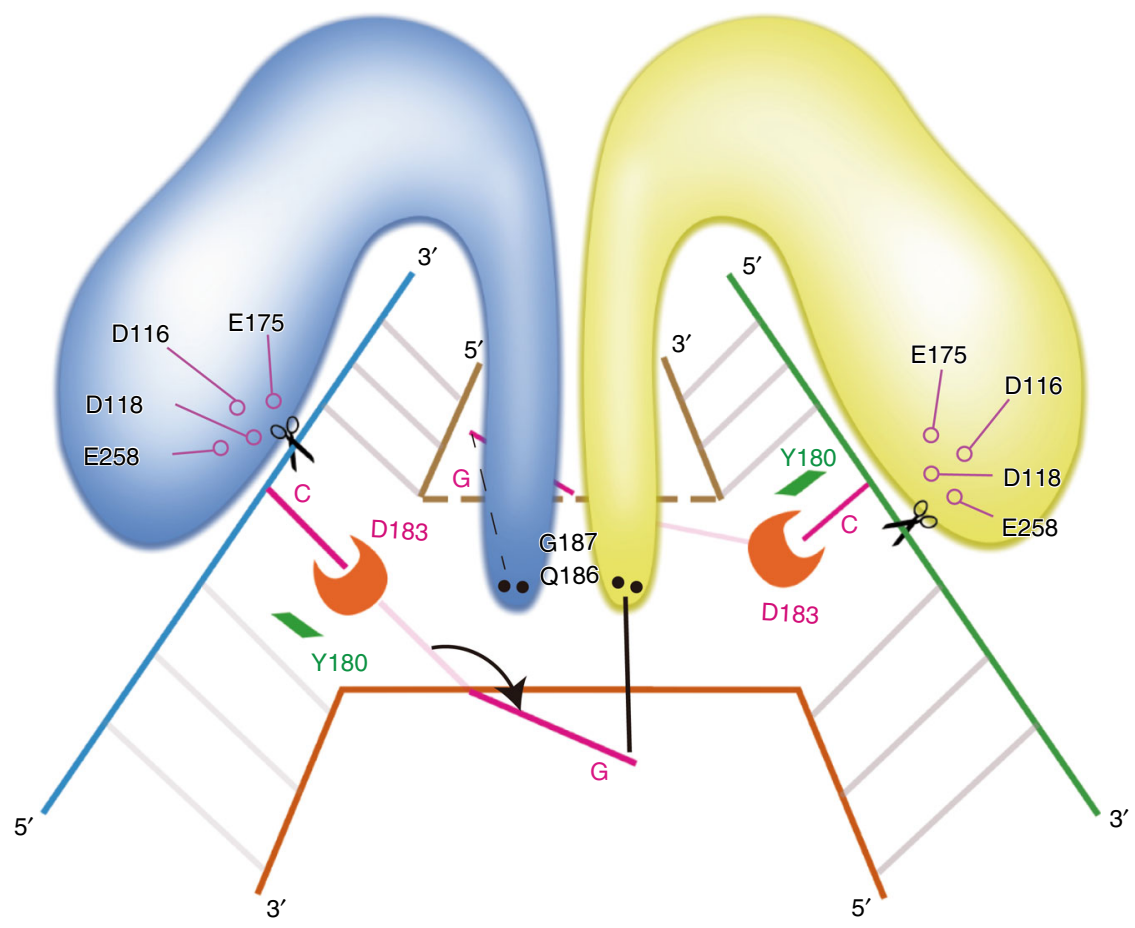

Fig. 6 Proposed working model for cytosine-dependent HJ resolution by NtMOC1. The blue and yellow models indicate the protein protomers. The dimeric enzyme penetrates into the junction, and the two residues $\mathrm{Y} 180$ and D183 from the BR loop protrude into the duplex and disrupt the base pairing at the crossover. Y180 intercalates into the bases, whereas D183 recognizes the cytosine prior to the cleavage site. The flipped-out guanine is further stabilized by residues from the N-terminal helical bundle (Q186 and G187). Four acidic residues constitute the catalytic tetrad, hydrolyzing the phosphodiester bond in a cytosine-dependent manner.

AATCG-3'; 5'-CCAGTGCCTTGCTGGGACATCTTTGCCC-3'; 5'-TAGACAG CTCCATCCAGCAAGGCACTGG- $\overline{3}^{\prime} ; 5^{\prime}$-CGATTACAACAGAGGATGGAGCTG TCTA-3'. The underlined nucleotides indicate the 2-bp homologous cores at the crossover) were resolved in lysis buffer $(25 \mathrm{mM}$ Tris- $\mathrm{HCl}, \mathrm{pH} 8.0,150 \mathrm{mM} \mathrm{NaCl})$ and mixed at an equimolar ratio and incubated in boiled water and gradually cooled to room temperature. Several X2 (CCGG) variants were derived similarly by mutation of the CCGG core sequences. To obtain co-crystals, a series of truncated HJs with varying arm length and CCGG core sequences were similarly annealed. The individual oligo sequences used for $\mathrm{HJ}$ annealing are deposited in Supplementary Table 3.

Crystallization. Crystallizations were performed using the sitting-drop vapor diffusion method at $18{ }^{\circ} \mathrm{C}$ by mixing equal volumes $(1 \mu \mathrm{l})$ of protein with the reservoir solution. For the apo MOC1, protein was concentrated to $10 \mathrm{mg} \mathrm{ml}^{-1}$ and a final concentration of $1 \mathrm{mM} \mathrm{MgCl}$ was added to the sample before crystallization trials. The rodlike crystals of ZmMOC1 (T107-V280) were grown in a well buffer containing $14 \%$ PEG3350, $0.15 \mathrm{M} \mathrm{NH}_{4} \mathrm{Cl}$ and grew to full size within 3 days. Highquality $\mathrm{ZmMOC1}$ crystals were soaked in the reservoir solution plus $0.1 \mathrm{M}$ 5-amino-2,4,6-triiodoisophthalic acid (I3C, Molecular Dimensions) for $2 \mathrm{~h}$. For NtMOC1, the tetra-mutant (N108-S275, I112V/Q162K/E235Q/E239Q) gave rise to crystals. The diamond shape crystal appeared overnight and grew to full size within three days in the well buffer containing $0.1 \mathrm{M}$ Bis-Tris, pH 6.3, 22\% PEG3350, $0.2 \mathrm{M} \mathrm{Li}_{2} \mathrm{SO}_{4}$

To obtain MOC1-HJ complex, a final concentration of $1 \mathrm{mM} \mathrm{MgCl}_{2}$ was added to the dimeric MOC1, which was further mixed with HJ DNA with a molar ratio of 1: 1.2. Numerous $\mathrm{HJs}$ with varying arm length and homologous cores were used to incubate with MOC1 to screen cocrystals. Most of the crystals of ZmMOC1-HJ complex were either poorly diffracted or showed no DNA density after data processing. After extensive and tedious trials, tetrahedron crystals from $\mathrm{HJ}$ with an arm length of $9 \mathrm{bp}$ in each direction with CATG homologous core sequences in complex with NtMOC1 (N108-S275, I112V/Q162K/E235Q/E239Q) were appeared in a buffer containing $0.1 \mathrm{M}$ Sodium acetate trihydrate, $\mathrm{pH}$ 5.0, 8.5\% PEG3350, 2\% Tacsimate, $\mathrm{pH}$ 4.0. And crystals of HJ with an arm length of 9-bp with CCGG homologous core sequence in complex with cleavage inactive $\mathrm{NtMOC1}$ mutant (N108-S275, I112V/Q162K/E235Q/239Q/D116A/E175A/D253A/E258A) were appeared in a buffer containing $0.1 \mathrm{M}$ sodium acetate trihydrate, $\mathrm{pH} 5.6,15 \%$ 3-Methyl-1,5-Pentane Diol, 2\% PEG4000, 2\% D-sorbitol. Crystals were harvested and flash-frozen in liquid nitrogen and cryoprotected by adding glycerol to a final concentration of $10-20 \%$. These crystals gave rise to good diffraction with good DNA density.
Data collection and structure determination. The diffraction data of I3C-soaked $\mathrm{ZmMOC} 1$ were collected by the in-house X-ray diffraction instrument (Rigaku). The diffraction data of $\mathrm{HJ}$-free and $\mathrm{HJ}$-bound $\mathrm{NtMOCl}$ were all collected at Shanghai Synchrotron Research Facility (SSRF) on beamline BL17U or BL19U. The data were integrated and processed with the HKL2000 program suite and XDS packages ${ }^{51}$. Further data processing was carried out using CCP4 suit ${ }^{52}$. Crystal structure of ZmMOC1 was determined at a resolution of $2.5 \AA$. The structure of apo and HJ-bound NtMOC1 were solved by molecular replacement using ZmMOC1 as search template. All the structures were iteratively built with $\mathrm{COOT}^{53}$ and refined with PHENIX program ${ }^{54}$. Data collection and structure refinement statistics are summarized in Supplementary Table 1. All figures were generated using the program PyMOL (http://www.pymol.org/).

Electrophoretic mobility shift assay (EMSA). Proteins were incubated with approximately $250 \mathrm{nM} \mathrm{HJ}$ substrates in a volume of $10 \mu \mathrm{l}$ containing the binding buffer (50 mM Tris-HCl, pH 8.0, $1 \mathrm{mM}$ DTT, $5 \mathrm{mM}$ EDTA, $200 \mathrm{ng} \mathrm{ml}^{-1}$ Heparin and $10 \%$ glycerol). After reaction for $30 \mathrm{~min}$ at $25^{\circ} \mathrm{C}$, the mixtures were resolved on $6 \%$ native acrylamide gels (37.5:1 acrylamide:bis-acrylamide) in $0.5 \times$ Trisglycine buffer under an electric field of $15 \mathrm{~V} \mathrm{~cm}^{-1}$ for $3 \mathrm{~h}$. Gels were extracted and stained by GelRed, and visualized by Image Lab (Bio-Rad). The results are representative of at least three independent experiments.

HJ cleavage assay. The cleavage reaction $(10 \mu \mathrm{l})$ was performed in the cutting buffer (50 mM Tris- $\mathrm{HCl}$, pH 8.0, $1 \mathrm{mM}$ DTT, $10 \mathrm{mM} \mathrm{MgCl}_{2}$ ). The final HJ concentration is $250 \mathrm{nM}$, and protein concentrations are gradually 2 -fold diluted from $1 \mu \mathrm{M}$. After $1 \mathrm{~h}$ reaction at $30^{\circ} \mathrm{C}$, the mixture was terminated by adding the termination buffer (50 mM Tris-Cl, pH 8.0, 3\% SDS, $50 \mathrm{mM}$ EDTA, Trypsin and pronase) and then incubated at $50^{\circ} \mathrm{C}$ for $10 \mathrm{~min}$. The reaction products were separated using $7 \%$ native PAGE. Gels were stained by GelRed, and visualized by Image Lab (Bio-Rad). The results are representative of at least three independent experiments.

Cleavage site mapping. The cleavage reaction $(10 \mu \mathrm{l})$ was performed in the cutting buffer ( $50 \mathrm{mM}$ Tris-Cl, $\mathrm{pH} 8.0,1 \mathrm{mM}$ DTT, $10 \mathrm{mM} \mathrm{MgCl}_{2}$ ). The $5^{\prime}$-end of one of the DNA strands $(1,2,3$ or 4$)$ was labeled with FAM. The final HJ concentration is $250 \mathrm{nM}$, and the protein concentration is $1 \mu \mathrm{M}$. The cleavage reaction was performed at $30^{\circ} \mathrm{C}$ for $1 \mathrm{~h}, 5 \mu \mathrm{l}$ of the digested $\mathrm{HJ}$ substrates were further mixed with $15 \mu \mathrm{l}$ of the denaturing buffer $(90 \%(\mathrm{v} / \mathrm{v})$ formamide, $20 \mathrm{mM}$ Tris-HCl, $\mathrm{pH} 8.0,20 \mathrm{mM}$ EDTA, $\mathrm{pH} 8.0,0.05 \%$ (w/v) bromophenol blue and $0.05 \%$ xylene cyanol), and denatured by incubating at $98^{\circ} \mathrm{C}$ for $10 \mathrm{~min}$. Then the 
reaction products were resolved on denaturing PAGE (23\% acrylamide, $7 \mathrm{M}$ urea, $1 \times \mathrm{TBE}, 1 \%(\mathrm{w} / \mathrm{v})$ APS and $0.1 \%$ TEMED). Fluorescent signals were detected by a scanner (Amersham Typhoon). To map the specific cleavage site, a series of $5^{\prime}$-FAM labeled oligos (12-17 nt) that include the homologous cores were used as markers.

Size exclusion chromatography (SEC). To evaluate the molecular mass of $\mathrm{NtMOC} 1$ and the relative mutants, proteins were subjected to SEC analysis (Superdex-200 Increase 10/300, GE Healthcare) using an AKTA FPLC instrument (GE healthcare) in a buffer containing $25 \mathrm{mM}$ Tris-HCl, $\mathrm{pH} 8.0,500 \mathrm{mM}$ $\mathrm{NaCl}$. Fractions with the same elution volume from each injection were subjected to SDS-PAGE and visualized by Comassie blue staining. The elution volume of the individual injections could be used to assess their molecular masses.

Analytical ultracentrifugation (AUC). The stoichiometry of MOC1 were investigated by AUC experiments, which was performed in a Beckman Coulter XL-I analytical ultracentrifuge using two-channel centrepieces. The proteins were in solutions containing $25 \mathrm{mM}$ Tris- $\mathrm{HCl}, \mathrm{pH} 8.0,500 \mathrm{mM} \mathrm{NaCl}$. Data were collected by absorbance detection at $18^{\circ} \mathrm{C}$ for proteins at a concentration of $0.8-1 \mathrm{mg} \mathrm{ml}^{-1}$ at a rotor speed of 45,000 r.p.m. The SV-AUC data were globally analyzed using the SEDFIT program and fitted to a continuous $c(s)$ distribution model to determine the molecular mass.

Reporting summary. Further information on research design is available in the Nature Research Reporting Summary linked to this article.

\section{Data availability}

The authors declare that all relevant data supporting the findings of this study are available within the article and its supplementary files or from the corresponding author upon reasonable request. The atomic coordinates and structure factors for the reported crystal structures have been deposited in the Protein Data Bank (PDB) with the accession codes $6 \mathrm{KVN}, 6 \mathrm{KVO}, 6 \mathrm{LCM}, 6 \mathrm{LCT}$. The source data underlying Figs. 1b, 3d, e, and 5b, c and Supplementary Figs 3b, c, e, f, 5d, e, 7a, b, 10c, d, and 12a, b are provided as a Source Data file.

Received: 14 September 2019; Accepted: 17 February 2020; Published online: 17 March 2020

\section{References}

1. Holliday, R. A mechanism for gene conversion in fungi. Genet. Res. Camb. 5, 282-304 (1964).

2. Lam, I. \& Keeney, S. Mechanism and regulation of meiotic recombination initiation. Cold Spring Harb. Perspect. Biol. 7, a016634 (2014).

3. Eichman, B. F., Vargason, J. M., Mooers, B. H. \& Ho, P. S. The Holliday junction in an inverted repeat DNA sequence: sequence effects on the structure of four-way junctions. Proc. Natl Acad. Sci. USA 97, 3971-3976 (2000).

4. Ortiz-Lombardia, M. et al. Crystal structure of a DNA Holliday junction. Nat. Struct. Biol. 6, 913-917 (1999).

5. Sarbajna, S. \& West, S. C. Holliday junction processing enzymes as guardians of genome stability. Trends Biochem. Sci. 39, 409-419 (2014).

6. Chan, Y. W. \& West, S. C. Spatial control of the GEN1 Holliday junction resolvase ensures genome stability. Nat. Commun. 5, 4844 (2014).

7. Wechsler, T., Newman, S. \& West, S. C. Aberrant chromosome morphology in human cells defective for Holliday junction resolution. Nature 471, 642-646 (2011).

8. Shah, R., Bennett, R. J. \& West, S. C. Genetic recombination in E. coli: RuvC protein cleaves Holliday junctions at resolution hotspots in vitro. Cell 79, 853-864 (1994).

9. Komori, K., Sakae, S., Shinagawa, H., Morikawa, K. \& Ishino, Y. A Holliday junction resolvase from Pyrococcus furiosus: functional similarity to Escherichia coli RuvC provides evidence for conserved mechanism of homologous recombination in Bacteria, Eukarya, and Archaea. Proc. Natl Acad. Sci. USA 96, 8873-8878 (1999).

10. Picksley, S. M., Parsons, C. A., Kemper, B. \& West, S. C. Cleavage specificity of bacteriophage T4 endonuclease VII and bacteriophage T7 endonuclease I on synthetic branch migratable Holliday junctions. J. Mol. Biol. 212, 723-735 (1990).

11. Ip, S. C. et al. Identification of Holliday junction resolvases from humans and yeast. Nature 456, 357-361 (2008).

12. Wyatt, H. D. \& West, S. C. Holliday junction resolvases. Cold Spring Harb. Perspect. Biol. 6, a023192 (2014).
13. Hadden, J. M., Declais, A. C., Carr, S. B., Lilley, D. M. \& Phillips, S. E. The structural basis of Holliday junction resolution by T7 endonuclease I. Nature 449, 621-624 (2007).

14. Biertumpfel, C., Yang, W. \& Suck, D. Crystal structure of T4 endonuclease VII resolving a Holliday junction. Nature 449, 616-620 (2007).

15. Bennett, R. J., Dunderdale, H. J. \& West, S. C. Resolution of Holliday junctions by RuvC resolvase: cleavage specificity and DNA distortion. Cell 74, 1021-1031 (1993).

16. Komori, K. et al. Biochemical characterization of the hic holliday junction resolvase of Pyrococcus furiosus. Nucleic Acids Res. 28, 4544-4551 (2000).

17. Shah Punatar, R., Martin, M. J., Wyatt, H. D., Chan, Y. W. \& West, S. C. Resolution of single and double Holliday junction recombination intermediates by GEN1. Proc. Natl Acad. Sci. USA 114, 443-450 (2017).

18. Liu, Y. et al. Crystal structure of a eukaryotic GEN1 resolving enzyme bound to DNA. Cell Rep. 13, 2565-2575 (2015).

19. Lee, S. H. et al. Human Holliday junction resolvase GEN1 uses a chromodomain for efficient DNA recognition and cleavage. Elife 4, e12256 (2015).

20. Bauknecht, M. \& Kobbe, D. AtGEN1 and AtSEND1, two paralogs in Arabidopsis, possess holliday junction resolvase activity. Plant Physiol. 166, 202-216 (2014).

21. Kleff, S., Kemper, B. \& Sternglanz, R. Identification and characterization of yeast mutants and the gene for a cruciform cutting endonuclease. EMBO J. 11, 699-704 (1992).

22. Oram, M., Keeley, A. \& Tsaneva, I. Holliday junction resolvase in Schizosaccharomyces pombe has identical endonuclease activity to the CCE1 homologue YDC2. Nucleic Acids Res. 26, 594-601 (1998).

23. Kobayashi, Y. et al. Holliday junction resolvases mediate chloroplast nucleoid segregation. Science 356, 631-634 (2017).

24. Fogg, J. M., Schofield, M. J., White, M. F. \& Lilley, D. M. Sequence and functional-group specificity for cleavage of DNA junctions by RuvC of Escherichia coli. Biochemistry 38, 11349-11358 (1999).

25. White, M. F. \& Lilley, D. M. The structure-selectivity and sequence-preference of the junction-resolving enzyme CCE1 of Saccharomyces cerevisiae. J. Mol. Biol. 257, 330-341 (1996).

26. Schofield, M. J., Lilley, D. M. \& White, M. F. Dissection of the sequence specificity of the Holliday junction endonuclease CCE1. Biochemistry 37, 7733-7740 (1998).

27. White, M. F. \& Lilley, D. M. Characterization of a Holliday junction-resolving enzyme from Schizosaccharomyces pombe. Mol. Cell Biol. 17, 6465-6471 (1997).

28. Whitby, M. C. \& Dixon, J. A new Holliday junction resolving enzyme from Schizosaccharomyces pombe that is homologous to CCE1 from Saccharomyces cerevisiae. J. Mol. Biol. 272, 509-522 (1997).

29. Gorecka, K. M., Komorowska, W. \& Nowotny, M. Crystal structure of RuvC resolvase in complex with Holliday junction substrate. Nucleic Acids Res. 41, 9945-9955 (2013).

30. Ariyoshi, M. et al. Atomic structure of the RuvC resolvase: a holliday junctionspecific endonuclease from E. coli. Cell 78, 1063-1072 (1994).

31. Raaijmakers, H. et al. X-ray structure of T4 endonuclease VII: a DNA junction resolvase with a novel fold and unusual domain-swapped dimer architecture. EMBO J. 18, 1447-1458 (1999).

32. Hadden, J. M., Convery, M. A., Declais, A. C., Lilley, D. M. \& Phillips, S. E. Crystal structure of the Holliday junction resolving enzyme T7 endonuclease I. Nat. Struct. Biol. 8, 62-67 (2001).

33. Ceschini, S. et al. Crystal structure of the fission yeast mitochondrial Holliday junction resolvase Ydc2. EMBO J. 20, 6601-6611 (2001).

34. Rafferty, J. B. et al. The structure of Escherichia coli RusA endonuclease reveals a new Holliday junction DNA binding fold. Structure 11, 1557-1567 (2003).

35. McGregor, N. et al. The structure of Bacillus subtilis RecU Holliday junction resolvase and its role in substrate selection and sequence-specific cleavage. Structure 13, 1341-1351 (2005).

36. Nishino, T., Komori, K., Ishino, Y. \& Morikawa, K. Dissection of the regional roles of the archaeal Holliday junction resolvase Hjc by structural and mutational analyses. J. Biol. Chem. 276, 35735-35740 (2001).

37. Bond, C. S., Kvaratskhelia, M., Richard, D., White, M. F. \& Hunter, W. N. Structure of $\mathrm{Hjc}$, a Holliday junction resolvase, from Sulfolobus solfataricus. Proc. Natl Acad. Sci. USA 98, 5509-5514 (2001).

38. Middleton, C. L., Parker, J. L., Richard, D. J., White, M. F. \& Bond, C. S. Substrate recognition and catalysis by the Holliday junction resolving enzyme Hje. Nucleic Acids Res. 32, 5442-5451 (2004).

39. Nowotny, M. Retroviral integrase superfamily: the structural perspective. EMBO Rep. 10, 144-151 (2009).

40. Holm, L. Benchmarking Fold Detection by DaliLite v.5. Bioinformatics 35 5326-5327 (2019)

41. Nishimasu, H. et al. Crystal structure of Cas9 in complex with guide RNA and target DNA. Cell 156, 935-949 (2014).

42. Yoshikawa, M., Iwasaki, H. \& Shinagawa, H. Evidence that phenylalanine 69 in Escherichia coli RuvC resolvase forms a stacking interaction during binding 
and destabilization of a Holliday junction DNA substrate. J. Biol. Chem. 276, 10432-10436 (2001).

43. Chen, L., Shi, K., Yin, Z. \& Aihara, H. Structural asymmetry in the Thermus thermophilus RuvC dimer suggests a basis for sequential strand cleavages during Holliday junction resolution. Nucleic Acids Res. 41, 648-656 (2013).

44. Klimasauskas, S., Kumar, S., Roberts, R. J. \& Cheng, X. HhaI methyltransferase flips its target base out of the DNA helix. Cell 76, 357-369 (1994).

45. Slupphaug, G. et al. A nucleotide-flipping mechanism from the structure of human uracil-DNA glycosylase bound to DNA. Nature 384, 87-92 (1996).

46. Zhou, R. et al. Junction resolving enzymes use multivalency to keep the Holliday junction dynamic. Nat. Chem. Biol. 15, 269-275 (2019).

47. Fogg, J. M., Kvaratskhelia, M., White, M. F. \& Lilley, D. M. Distortion of DNA junctions imposed by the binding of resolving enzymes: a fluorescence study. J. Mol. Biol. 313, 751-764 (2001).

48. White, M. F. \& Lilley, D. M. Interaction of the resolving enzyme YDC2 with the four-way DNA junction. Nucleic Acids Res. 26, 5609-5616 (1998).

49. Gorecka, K. M. et al. RuvC uses dynamic probing of the Holliday junction to achieve sequence specificity and efficient resolution. Nat. Commun. 10, 4102 (2019).

50. Lin, H. et al. Structural basis of sequence-specific Holliday junction cleavage by MOC1. Nat. Chem. Biol. 15, 1241-1248 (2019).

51. Otwinowski, Z. \& Minor, W. Processing of X-ray diffraction data collected in oscillation mode. Methods Enzymol. 276, 307-326 (1997).

52. Winn, M. D. et al. Overview of the CCP4 suite and current developments. Acta Crystallogr. D. Biol. Crystallogr. 67, 235-242 (2011).

53. Emsley, P. \& Cowtan, K. Coot: model-building tools for molecular graphics. Acta Crystallogr. D. 60, 2126-2132 (2004).

54. Adams, P. D. et al. PHENIX: building new software for automated crystallographic structure determination. Acta Crystallogr. D. Biol. Crystallogr. 58, 1948-1954 (2002).

\section{Acknowledgements}

We thank the staff of the BL17U1/BL19U1/BL19U2 beamline of the NCPSS at the Shanghai Synchrotron Radiation Facility for assistance during data collection; and research associates at the Center for Protein Research, Huazhong Agricultural University, for technical support. This work was supported by funds from the Ministry of Science and Technology of China (2018YFA0507700), the National Natural Science Foundation of China ( 31700203 for J.Y. and 31722017 for P.Y.), the Fork Ying-Tong Education Foundation (151021), the Fundamental Research Funds for the Central Universities (2662017PY031), and the China Postdoctoral Science Foundation (2017T100561).

\section{Author contributions}

J.Y., S.H., and P.Y. designed all experiments. J.Y., S.H., and W.H. performed protein purification and crystallization. Z.G. determined all the structures. J.Y., S.H., and D.Z. performed the biochemical assays. All authors analyzed the data and contributed to paper preparation. J.Y. and P.Y. wrote the paper.

\section{Competing interests}

The authors declare no competing interests.

\section{Additional information}

Supplementary information is available for this paper at https://doi.org/10.1038/s41467020-15242-8.

Correspondence and requests for materials should be addressed to P.Y.

Peer review information Nature Communications thanks Anne-Cécile Déclais, Takehiko Shibata and the other, anonymous, reviewer(s) for their contribution to the peer review of this work. Peer reviewer reports are available.

Reprints and permission information is available at http://www.nature.com/reprints

Publisher's note Springer Nature remains neutral with regard to jurisdictional claims in published maps and institutional affiliations.

(c) (i) Open Access This article is licensed under a Creative Commons Attribution 4.0 International License, which permits use, sharing, adaptation, distribution and reproduction in any medium or format, as long as you give appropriate credit to the original author(s) and the source, provide a link to the Creative Commons license, and indicate if changes were made. The images or other third party material in this article are included in the article's Creative Commons license, unless indicated otherwise in a credit line to the material. If material is not included in the article's Creative Commons license and your intended use is not permitted by statutory regulation or exceeds the permitted use, you will need to obtain permission directly from the copyright holder. To view a copy of this license, visit http://creativecommons.org/ licenses/by/4.0/.

(C) The Author(s) 2020 